\title{
Common processes underlie enhanced recency effects for auditory and changing-state stimuli
}

\author{
ARTHUR M. GLENBERG \\ University of Wisconsin, Madison, Wisconsin
}

\begin{abstract}
For some stimuli, dynamic changes are crucial for identifying just what the stimuli are. For example, spoken words (or any auditory stimuli) require change over time to be recognized. Kallman and Cameron (1989) have proposed that this sort of dynamic change underlies the enhanced recency effect found for auditory stimuli, relative to visual stimuli. The results of three experiments replicate and extend Kallman and Cameron's finding that dynamic visual stimuli (that is visual stimuli in which movement is necessary to identify the stimuli), relative to static visual stimuli, engender enhanced recency effects. In addition, an analysis based on individual differences is used to demonstrate that the processes underlying enhanced recency effects for auditory and dynamic visual stimuli are substantially similar. These results are discussed in the context of perceptual grouping processes.
\end{abstract}

Do stimuli that undergo dynamic transformations lead to improved memory? That is the claim of the Campbell and Dodd (1980) changing-state hypothesis: The enhanced recall of recently presented auditory information, compared with the recall of recently presented visual information, is due to the dynamic nature of spoken words. Kallman and Cameron (1989) generated support for this hypothesis using a clever comparison. Subjects recalled sequences of directions (e.g., up, down, left, right) conveyed by a static orthographic presentation of words (e.g., "up") or by a dynamic visual stimulus that moved in the appropriate direction (a box, presented on a computergenerated display, that moved up, down, left, or right). Kallman and Cameron found an enhanced recency effect in this moving-box condition.

The research reported here asks (and answers) three questions about Kallman and Cameron's effect. First, can the effect be replicated? (Yes.) Second, if the effect is replicated, can it be extended using a variant of their procedure? (Yes.) Third, are the processes that produce the enhanced recency effect for dynamic visual stimuli the same as the processes that produce enhanced recency effects for auditory stimuli? (Yes.)

This article has the following organization. The remainder of the introduction presents a brief review of tests of the Campbell and Dodd changing-state hypothesis, and a consideration of what it takes to demonstrate that the same processes contribute to two empirical effects (e.g.,

\footnotetext{
This research was funded by National Science Foundation Grant BNS 8416300 and University of Wisconsin Graduate School Grant 890200 awarded to the author. Thanks are due Ronald Barnhart and David Bensinger for their contributions to this research, and William Epstein, Margaret Intons-Peterson, Howard Kallman, and James Naime for their comments on an earlier draft of this paper. Requests for reprints may be sent to Arthur Glenberg, Department of Psychology, 1202 West Johnson St., Madison, Wisconsin 53706.
}

do the same processes contribute to the auditory recency effect and the moving-box recency effect?). Following the introduction are four experiments that provide the answers to the questions listed above. The General Discussion contains some far-fetched, but interesting, speculation relating recency and modality effects to perceptual grouping processes.

The modality effect is generally defined as the enhanced recall of recently presented auditory information, compared with the recall of recently presented visual information. Reviews of the literature are provided by Nairne (1988) and Penney $(1975,1989)$. A number of explanations for the effect have been proposed, but, for now, the focus will be on the changing-state hypothesis. Initial support for the hypothesis came from the demonstration that the recency effect for lip-read words (in which the visual stimulus changes state) is more similar (in size) to memory for auditory words than to memory for orthographic stimuli (Campbell \& Dodd, 1980). As Kallman and Cameron (1989) point out, however, a number of other explanations of the modality effect can account for this finding.

Crowder (1986) attempted several direct tests of the changing-state hypothesis. For example, in one experiment orthographically presented words moved across a computer screen, thereby changing state. In other experiments, the words were presented letter by letter. None of the manipulations enhanced recall of the visual stimuli, leading Crowder to conclude that the changing-state hypothesis was disconfirmed.

In contrast, Kallman and Cameron (1989) appear to have uncovered a critical variable that controls when changing-state stimuli enhance recall. They proposed that changes in the stimulus enhance memory only when the changes are used in identifying the stimulus. Thus, the dynamic changes in a spoken word or a lip-read word are essential to identifying the particular word, and these changes enhance memory (compared with memory in- 
duced by a static, orthographic presentation of a word). Moving an orthographically presented word, as in Crowder's (1986) experiments, does not contribute to the word's identification, and so no improvement in memory is to be expected.

Kallman and Cameron (1989) tested their proposal using a serial recall paradigm, in which subjects attempted to recall lists of seven directions. In the orthographic condition, the lists were composed of the stimuli "up," "down," "left," and "right" presented centered on a computer screen. Each stimulus in the moving-box condition consisted of a rectangle, initially presented in the center of the screen, which then moved in one of the four directions. Note that, in this condition, the movement is necessary to identify the stimulus. The most interesting finding was that the moving-box condition produced a small, but statistically significant, enhancement of the recency effect, compared with that produced by the orthographic condition.

Experiment 1 was designed to achieve two goals. The first was to replicate the finding of an enhanced recency effect in a condition comparable to Kallman and Cameron's moving-box condition. The second goal was to determine if the cognitive processes that produce the recency effect in the moving-box condition are the same as the processes that produce the recency effect for auditory stimuli. That is, the moving-box recency effect may represent a new, unrelated finding that in itself needs explaining. Alternatively, there may be only one recency effect (that is, one set of processes that produces recency effects). In this case, the moving-box version and the auditory version of the effect should mutually constrain explanations.

Determining whether or not the same cognitive processes contribute to two empirical phenomena is a tricky enterprise. The standard methodology has been to search for various types of dissociations. Thus, if an independent variable has an effect in one task and an opposite effect in another task, it would seem that the two tasks tap different processes. Dunn and Kirsner (1988) discuss a number of flaws in this reasoning, however, and they propose a new method they call reversed association. To use this method, performance in one task (e.g., recall of auditory stimuli) is plotted as a function of performance in the other task (e.g., recall of moving-box stimuli) across at least three levels of some other variable (e.g., serial position). According to Dunn and Kirsner's analysis, if the resulting function is nonmonotonic, the two tasks must have some processes that are not in common. Unfortunately, the reversed-association method has several drawbacks. First, a monotonic function does not guarantee that the tasks tap the same processes. Second, because most memory tasks tap multiple processes, some of which may be common to two tasks and some unique, the reversed-association procedure may produce nonmonotonic functions even when the two tasks do share some processes of interest.

Another method of determining if two phenomena tap the same process(es) uses a correlational analysis. The analysis is based on the following logic. First, suppose that there are stable individual differences in efficiency (e.g., speed, accuracy, or capacity) of cognitive processes. Second, suppose that performance is a function of the efficiencies of the cognitive processes contributing to a task. It follows, then, that if two tasks tap the same processes, performance on the two tasks will be correlated. This procedure must be used with some caution because the correlation can be affected by various statistical artifacts and general performance level. Precautions taken to avoid these problems are described in the results section. Variants of this procedure are discussed by Crowder (1982), Glenberg, Jona, and Curran (1989), and Underwood (1975).

\section{EXPERIMENT 1}

Experiment 1 was modeled after Kallman and Cameron's (1989) study. Each trial required subjects to serially recall seven directional stimuli (up, down, left, and right). Each subject participated in four different presentation conditions, although all stimuli on a particular trial were in the same presentation condition. In the auditory condition, the stimuli were reconstructed from digitized exemplars of the spoken direction words. In the orthographic condition, each stimulus word was presented in the middle of a video display screen. For each stimulus in the moving-box condition, a rectangle was presented in the middle of the screen, and it appeared to move in one of the four directions. Finally, in the stationary-box condition, for each stimulus a rectangle was presented in a location displaced up, down, left, or right of a fixation point, but the box did not move during its presentation.

On the basis of past results, the expectation is that the largest recency effect will be found in the auditory condition. If the Kallman and Cameron result replicates, then the moving-box condition will produce the next largest recency effect, and that recency effect should be larger than the effects produced by the orthographic and stationarybox conditions. Furthermore, if the moving-box recency effect and the auditory recency effect are tapping the same cognitive processes, then the recency effects from these two tasks will correlate significantly. Finally, the correlation between the auditory recency effect and the movingbox recency effect should be larger than the correlation of the auditory recency effect with either the orthographic recency effect or the stationary-box recency effect.

\section{Method}

Subjects. The subjects were 24 undergraduates ( 9 men, 15 women) from the University of Wisconsin-Madison who participated in the study to receive extra credit in an introductory psychology course. Experimental participation was one option for acquisition of the extra credit.

Design and Apparatus. All events in the experiment were timed and controlled by an Apple Macintosh II computer and an Apple AppleColor monitor.

The subjects participated in all four presentation conditions, receiving 20 trials (plus practice) in each condition. The order of the four presentation conditions was counterbalanced over subjects. 
The structure of a trial was identical in each condition. The trial began with a ready signal (e.g., "Get ready for auditory words") presented on the monitor for $1,000 \mathrm{msec}$. The ready signal always indicated the modality of the upcoming trial. Next, seven stimuli chosen from the set of direction terms ("up," "down," "left," and "right") were presented. The stimuli were randomly chosen, except for the constraint that each occur once in the first four serial positions. Each stimulus was presented for $700 \mathrm{msec}$. There was a 300-msec pause between each stimulus, during which time the screen was blank. In the auditory condition, the stimuli were created by digitizing speech at a rate of 11,264 samples per second and converting each sample into 1 of 128 values. On playback using the speaker built into the Macintosh, the digitized words were between 300 and $400 \mathrm{msec}$ in duration. An extra pause was inserted after each stimulus so that the total stimulus onset asynchrony was, as in the other conditions, $1,000 \mathrm{msec}$. In the orthographic condition, the direction words appeared, one at a time, $0.5 \mathrm{~cm}$ above a fixation point in the center of the monitor. In the stationary-box condition, each stimulus consisted of a $1.8 \mathrm{~cm} \times 1.8 \mathrm{~cm}$ box that appeared about $2 \mathrm{~cm}$ above, below, to the left of, or to the right of the fixation point. In the moving-box condition, boxes of the same dimensions started in the center of the screen and moved about $2 \mathrm{~cm}$ up, down, to the left, or to the right. The boxes moved during the 700 -msec presentation time only.

The response method was the same for all of the conditions. After the last stimulus of a trial was presented, seven dashes appeared on the monitor screen. Each time a subject pressed the up, down, left, or right arrow key on the keyboard, one of the dashes was replaced with a letter ( $U, D, L$, or $R$ ) to recreate the sequence. The arrow keys were contiguous and were arranged on the keyboard in a pattern analogous to the directions. That is, the up arrow key was the topmost, the down arrow key was directly below it, and the left and right arrow keys were to the left and right of the down arrow key, respectively. This arrangement should promote stimulus-response compatibility. Responding was self-paced. If a subject believed that he or she had made a mistake, the subject could delete and reenter the responses. When satisfied with his or her response, the subject pressed an end key to register the responses and present the next trial.

Procedure. The subjects participated individually. Each subject provided written consent before participating in the experiment. The subject was seated approximately $50 \mathrm{~cm}$ from the monitor screen in a soundproof chamber. During the instruction period, the subject viewed one example of each of the presentation conditions and then completed four practice trials in the first presentation condition. The first block of 20 trials followed. Before each successive block of 20 trials, the subjects participated on two trials using the presentation condition of the upcoming block.

\section{Results}

The number of stimuli correctly recalled (out of 20) were counted for each serial position. Two types of analyses will be presented. First, analyses of variance on the mean recall will be discussed. These analyses determine whether or not there is an enhanced recency effect in the moving-box condition. The second set of analyses are designed to determine if the moving-box and auditory recency effects are produced by the same mechanisms.

Figure 1 illustrates the serial-position curves in the four presentation conditions. As is typical, there were strong serial-position effects, including a recency effect (improvement in recall between Serial Positions 6 and 7). Also as is typical, the recency effect was more pronounced for auditory presentation than for orthographic presentation. There was also a hint that the recency effect in the moving-box condition was slightly larger than was the effect in the orthographic and stationary-box conditions. Statistically, the main effect of presentation condition was not significant (using a Type I error rate of .05 , as in all analyses reported), but the main effect for serial position was $\left[F(6,138)=33.91, M S_{e}=9.09\right]$. Also, the interaction between presentation condition and serial position was significant $\left[F(18,414)=4.15, M S_{\mathrm{e}}=2.78\right]$.

Planned comparisons were used to test for specific effects. First, the recency effect (difference in recall between Serial Positions 6 and 7) in the auditory condition exceeded the recency effect in the other conditions

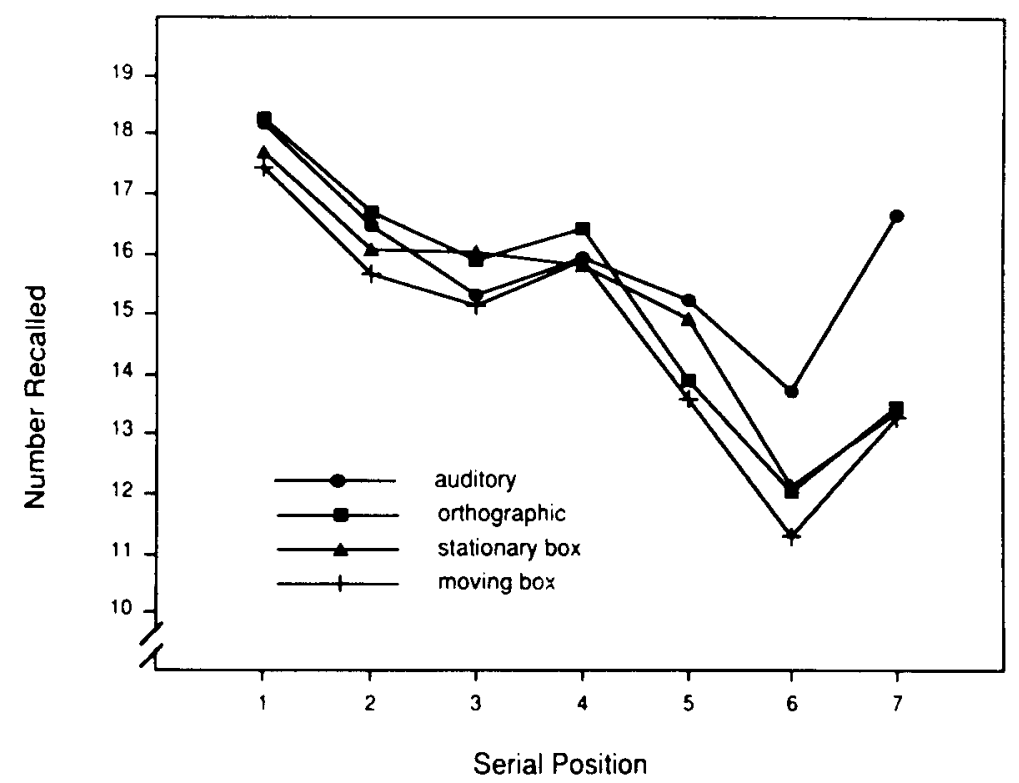

Figure 1. Number recalled from each serial position in Experiment 1. 
$\left[F(1,23)=5.49, M S_{\mathrm{e}}=3.04\right]$. Second, the recency effect for the moving-box condition was significant $[F(1,23)=$ $23.49, M S_{\mathrm{e}}=2.041$. However, the recency effect for the moving-box condition was not significantly greater than the recency effects in the orthographic and stationary-box conditions $\left[F(1,23)=1.92, M S_{\mathrm{e}}=1.85\right]$. Thus, considering this result alone, there is little statistical evidence for an enhanced recency effect in the moving-box condition. It is likely that this is a Type II error. Results from Experiments 2 and 3 will demonstrate statistical support for an enhanced recency effect in the moving-box condition. Furthermore, the results from this experiment and Experiments 2 and 3 will support the conclusion that the recency effect in the moving-box condition is different in kind from the recency effect in the orthographic and stationary-box conditions. Thus, the conclusion that there is an enhanced recency effect in the moving-box condition is likely to be correct, regardless of the outcome of this specific comparison.

The second set of analyses was designed to determine if the recency effect in the moving-box condition is produced by the same processes as is the recency effect in the auditory condition. The basic idea is to correlate a measure of the size of the recency effect (corrected for overall level of performance) in the two conditions. A significant correlation is taken as evidence that the same processes are contributing to the two recency effects.

A commonsense measure of the size of the recency effect is the difference in recall between Serial Positions 6 and 7 , as used above. Unfortunately, the difference score is plagued with problems. First, the difference score is less reliable than either of the components entering into it (Cohen \& Cohen, 1975), thus reducing the size of any correlations. Second, although the difference score seems to be a measure of improvement corrected for overall level of performance (by subtraction of recall at Position 6), in most cases, the simple difference is an overcorrection (Cohen \& Cohen, 1975). Third, the correlation of two difference scores may be grossly affected by their joint correlation with overall performance (Chapman \& Chapman, 1988).

Fortunately, there is a measure of the size of recency that overcomes these problems. This score is recall at Serial Position 7, with recall of Serial Position 6 partialed out. The score is formed by calculating the regression equation, using recall at Serial Position 6 as the predictor variable and recall at Serial Position 7 as the dependent variable. This equation is used to predict, for each subject, the subject's recall at Serial Position 7, using his/her recall at Position 6 as the predictor variable. This predicted recall is then subtracted from the actual recall at Serial Position 7, giving a partialed, or residual, score. This partialed score will be referred to as the recency score.

Consider what this recency score represents. Virtually all theories of recency effects assume that something different, an extra process or an extra representation, is affecting the encoding, storage, or retrieval of information presented at the end, rather than the middle, of a list.
Table 1

Correlations Among the Recency Scores in Experiment 1

\begin{tabular}{|c|c|c|c|c|}
\hline & Auditory & Orthographic & Moving Box & $\begin{array}{c}\text { Stationary } \\
\text { Box }\end{array}$ \\
\hline Auditory & & .31 & $.40^{*}$ & .15 \\
\hline Orthographic & & & .28 & .21 \\
\hline Moving Box & & & & .23 \\
\hline
\end{tabular}

To the extent that the efficiency of the extra process is not perfectly correlated with processes contributing to recall of the middle items, the partialed score is a measure of the contribution of the extra process to recall.

For the sake of argument, assume that the extra process is highly correlated with processes contributing to recall of the middle items. In that case, the partialed score would reflect only noise, and there would be no significant correlations. The significant correlations (discussed shortly) found in these experiments allow us to discount this possibility.

Now assume that an extra process does contribute to recency effects, but that the process contributes to all recency effects. In that case, recency scores in all of the conditions should correlate equally well. That was not the case, however, indicating that different processes contribute to different recency effects.

A recency score was formed for each subject in each condition. The correlations among the recency scores are presented in Table 1. Note that the auditory recency score and the moving-box recency score are significantly correlated, whereas the auditory recency score does not correlate significantly with any of the other recency scores. This finding can be interpreted as follows: The processes that generate the auditory recency effect have a substantial overlap with the processes that generate the moving-box recency effect.

Less weight is given to the other correlations, even those close in size to the significant one, because they are not consistent across the three experiments reported here. Comparisons across the experiments are discussed in the Subsidiary Analyses section, following the discussion of Experiment 3.

\section{EXPERIMENT 2}

The data from Experiment 1 are not as strong statistically as one would like. In particular, there is little evidence that the size of the moving-box recency effect is much greater than the size of the orthographic or the stationary-box recency effect. Also, the correlation between the moving-box and auditory recency scores is just barely significant. Thus, replication seems in order.

A second reason for Experiment 2 is that there is a possibility that the correlation between the moving-box and auditory recency scores may have little to do with the dynamic quality of the moving-box stimuli. In particular, the moving-box condition can be quite difficult, and subjects may mouth or whisper the direction words in the 
moving-box condition in an effort to enhance memory. ${ }^{1}$ Other research (e.g., Nairne \& Walters, 1983) has demonstrated that mouthing produces effects very similar to those found with the auditory modality. Thus, the correlation between the moving-box and auditory conditions may reflect nothing more than subjects' turning the movingbox condition into an auditory-like condition by mouthing. In Experiment 2, mouthing was prevented by requiring articulatory suppression throughout the presentation of stimuli in all of the conditions.

\section{Method}

The method and design of Experiment 2 were identical to those of Experiment 1, except in two details. First, 36 subjects (19 men, 17 women), selected from the same source as those in Experiment 1, were used. Second, the subjects were instructed to engage in articulatory suppression. On each trial, starting with the ready signal and ending with the recall signal, the subjects repeated out loud the word "the" two to three times per second.

\section{Results}

The means of recall at each serial position are presented in Figure 2. There was a main effect of presentation $\left[F(3,105)=2.68, M S_{\mathrm{e}}=36.51\right]$. Also, there was a main effect of serial position $\left[F(6,210)=39.41, M S_{\mathrm{e}}=8.93\right]$. The interaction between presentation condition and serial position was also significant $\left[F(18,630)=2.70, M S_{e}=\right.$ 4.56]. Components of this interaction, as revealed by planned comparisons, are of most interest.

The recency effect (improvement in recall from Position 6 to Position 7) in the auditory condition exceeded the recency effect in the other conditions $[F(1,35)=$ 13.52, $\left.M S_{e}=3.46\right]$. Also, the recency effect in the moving-box condition was significant $[F(1,35)=28.58$, $M S_{\mathrm{e}}=3.43$ ]. In contrast to the data from Experiment 1, the recency effect in the moving-box condition was larger than the recency effect in the stationary-box and orthographic conditions $\left[F(1,35)=6.55, M S_{\mathrm{e}}=2.15\right]$. Thus, there is evidence for an enhanced recency effect in the dynamic moving-box condition.

Table 2 contains the correlations among the conditions using the recency scores. As in Experiment 1, the movingbox condition is the only condition to correlate significantly with the auditory condition. The moving-box recency scores were also significantly correlated with the orthographic recency scores. However, this correlation is not found consistently across the experiments.

The results from Experiment 2 are important in three respects. First, they confirm that there is an enhanced recency effect in the moving-box condition, thereby adding statistical support to the finding from Experiment 1 and replicating Kallman and Cameron (1989). Second, the important correlational results from Experiment 1 were also replicated. Apparently, the processes that produce the auditory recency effect are substantially similar to those that produce the moving-box recency effect. Also, whatever it is that produces the auditory recency effect (see General Discussion), it is more closely related to processes active in the moving-box condition than to processes active in the stationary-box condition and the orthographic condition. Third, because the subjects were engaged in articulatory suppression, it is unlikely that covert articulations in the moving-box condition were the cause of the enhanced moving-box recency effect and the correlation with the auditory condition.

\section{EXPERIMENT 3}

Kallman and Cameron (1989) have proposed that the critical feature that produces the enhanced recency effect in the moving-box condition (and in auditory conditions)

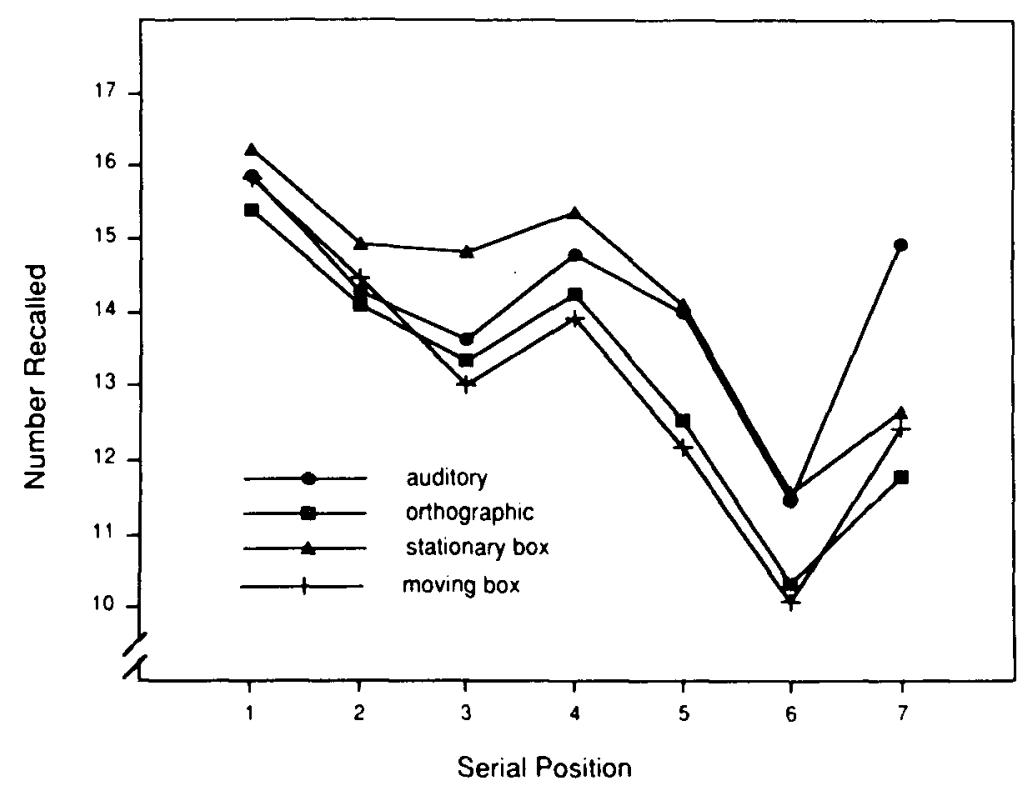

Figure 2. Number recalled from each serial position in Experiment 2. 
Table 2

Correlations Among the Recency Scores in Experiment 2

\begin{tabular}{|c|c|c|c|c|}
\hline & Auditory & Orthographic & Moving Box & $\begin{array}{c}\text { Stationary } \\
\text { Box }\end{array}$ \\
\hline Auditory & & .28 & $.43^{*}$ & -.02 \\
\hline Orthographic & & & $.43^{*}$ & .03 \\
\hline Moving Box & & & & .18 \\
\hline
\end{tabular}

${ }^{*} p<.01$

is that dynamic information is crucial in identifying the stimuli. Experiment 3 provides converging evidence for this proposal by using a different task in which dynamic information is used in identifying the stimuli. In this experiment, the seven-item lists were composed of three speed terms: fast, medium, and slow. In the auditory condition, the terms were presented by digitized speech. In the orthographic condition, the words were presented on the monitor screen. In the moving-box condition, the box appeared to move across the screen at a fast, medium, or slow rate. Thus, the rate of movement was used in identifying the stimulus. In the stationary-box condition, the words "fast," "medium," and "slow" were presented on the screen, and a box appeared under the appropriate word for the duration of the stimulus. Support for Kallman and Cameron's position will take the form of an enhanced recency effect in the moving-box condition. Additionally, we might expect a larger correlation between the auditory and moving-box conditions than between the auditory condition and the other conditions.

\section{Method}

The method was similar to that used in the previous experiments, except for the following differences. First, data were collected from a new group of 24 subjects ( 12 men, 12 women). Second, the stimuli were the three speed terms: fast, medium, and slow. In the audi- tory condition, the speech was digitized at a rate of 22,528 samples per second; on playback, each stimulus was exactly $500 \mathrm{msec}$ in duration. In the box conditions, the size of the box was decreased to approximately $.5 \mathrm{~cm}$ a side. In the moving-box condition, the box moved during the $700-\mathrm{msec}$ stimulus duration from left to right approximately 5,10 , and $20 \mathrm{~cm}$, to indicate the fast, medium, and slow stimuli, respectively. In the stationary-box condition, the words "fast," "medium," and "slow" were on the display screen throughout the trial. These words were located at approximately 5, 10, and $20 \mathrm{~cm}$ from the left edge of the display monitor. To indicate a speed, the box appeared under the appropriate word for the $700-\mathrm{msec}$ duration of the stimulus.

\section{Results}

Mean recall is illustrated in Figure 3. There were significant main effects of presentation condition $[F(3,69)=$ 9.31, $\left.M S_{\mathrm{e}}=10.46\right]$ and serial position $[F(6,138)=$ 31.30, $\left.M S_{\mathrm{e}}=6.21\right]$. The interaction was also significant $\left[F(18,414)=3.50, M S_{\mathrm{e}}=2.43\right]$

As in the previous experiments, the planned contrasts are of most interest. First, the auditory recency effect was significantly larger than the others $[F(1,23)=8.00$, $\left.M S_{\mathrm{e}}=4.25\right]$. Second, the recency effect in the movingbox condition was significant $\left[F(1,23)=16.82, M S_{\mathrm{e}}=\right.$ $2.19]$. Third, the recency effect in the moving-box condition was significantly greater (at the .06 level) than the recency effect in the orthographic and stationary-box conditions $\left[F(1,23)=3.75, M S_{e}=1.20\right]$. Thus, the data indicate a larger recency effect with dynamic visual stimuli than with static visual stimuli.

Table 3 contains the results of the correlational analyses of the recency scores. Again, the auditory recency scores correlate most highly with the moving-box recency scores. Thus, these data support the claim that the processes that contribute to the auditory recency effect also contribute to the moving-box recency effect much more than to the stationary-box recency effect. It now

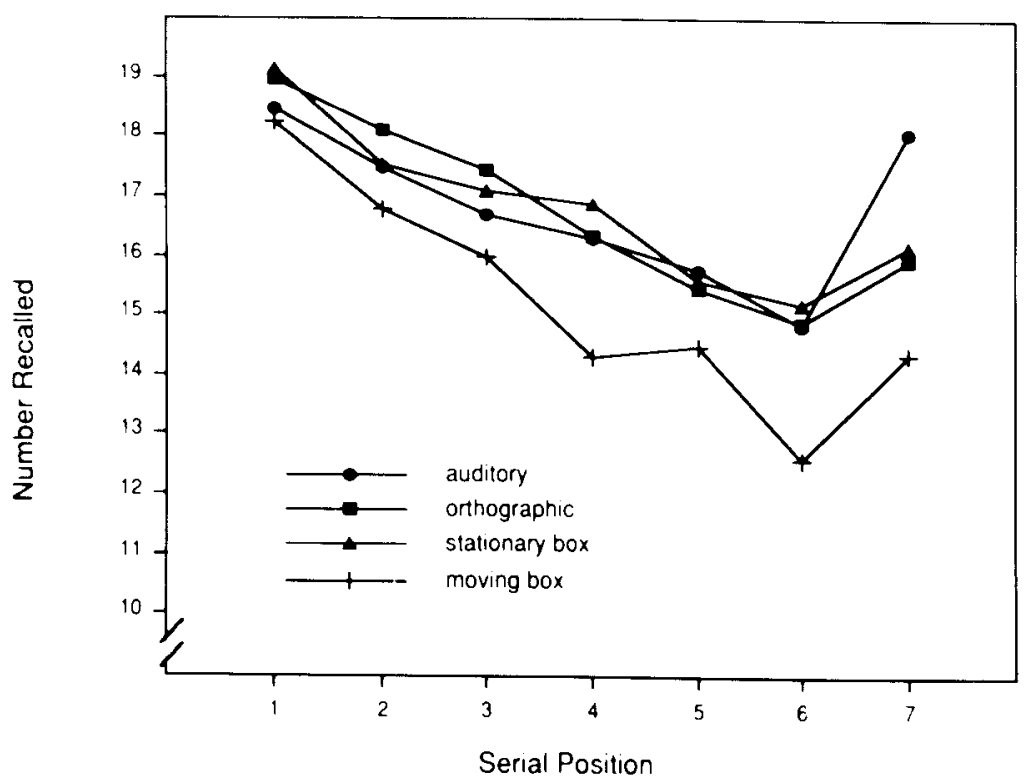

Figure 3. Number recalled from each serial position in Experiment 3. 
Table 3

Correlations Among the Recency Scores in Experiment 3

\begin{tabular}{|c|c|c|c|c|}
\hline & Auditory & Orthographic & Moving Box & $\begin{array}{c}\text { Stationary } \\
\text { Box }\end{array}$ \\
\hline Auditory & & .38 & $.43^{*}$ & .01 \\
\hline Orthographic & & & .25 & .31 \\
\hline Moving Box & & & & $.41^{*}$ \\
\hline
\end{tabular}

seems to be a good bet that some process sensitive to dynamic information is contributing to both effects.

\section{SUBSIDIARY ANALYSES FOR EXPERIMENTS 1-3}

Arguments are presented in the results section of Experiment 1 for using the recency score. In the interest of checking on those arguments, a number of other analyses were conducted and are reported in this section. In addition, an analysis is reported to determine if the process common to the auditory and moving-box conditions is specific to the recency portion of the serial-position curve.

Whenever multiple analyses are conducted, there is the danger of making Type I errors more frequently than is indicated by the nominal Type I error rate. When the analyses are distributed across three experiments, that danger would seem to be enhanced even further. Fortunately, having three similar experiments also provides an alternative method of assessing the reliability of a finding, namely its consistency across the three experiments. Consider the data presented in Tables 1-3. The correlation between the auditory and moving-box conditions is completely consistent in that it is significant in all three experiments. Although two other conditions also correlate significantly (orthographic and moving-box conditions in Experiment 2; moving-box and stationary-box conditions in Experiment 3), neither of these effects is consistent in that neither is significant in more than one experiment. To limit Type I errors, this notion of consistency, in addition to standard levels of significance, will be applied to the following analyses.

One alternative to the recency score is recall in the last serial position. A problem with using the last serial position as an index of recency is that correlations across the conditions will reflect individual differences in general memory ability (i.e., good subjects will tend to recall more than will poor subjects in all conditions). Thus, we would expect many significant and consistent correlations, but they may indicate nothing more than stable individual differences in general memory ability. Consistent with this reasoning, of the six pairs of conditions, five correlated significantly and consistently (that is, in at least two of the three experiments). The one pair that never produced a significant correlation was the auditory and the stationarybox condition.

To correct for general memory ability, one could subtract from recall at the last serial position recall at the penultimate position. As noted in the results section of
Experiment 1, this correction is less than optimal for a number of reasons-one of which is that the resulting difference score is not as reliable as either of the individual scores. If reliability is a problem, then the correlations may be inconsistent across the experiments. In fact, using the difference in recall between Serial Positions 6 and 7 as the dependent variable, four pairs of conditions correlated significantly, but none was consistent. That is, no pair of conditions produced a significant correlation in more than one experiment.

Another measure of the size of the recency effect (corrected for overall level of performance) is the proportion of all errors that occur at the last serial position (cf. Nairne and Walters, 1983). ${ }^{2}$ I will refer to this as the error score. Like the simple difference, the error score will tend to be less reliable than recall at any specific position. The problem is that the error score reflects variability in the last serial position, as well as variability in all of the other serial positions. Thus, we are led to expect low and inconsistent correlations. Using the error score as the dependent variable, most of the correlations were low (in fact, five were negative); however, one correlation was significant in two of the three experiments. The correlations between the stationary-box and moving-box conditions were .76, .67, and .10 in Experiments 1, 2, and 3, respectively. It is uncertain as to why this correlation is substantial in two experiments and not even close to significant in the third.

Up to this point, the analyses have been guided by the question of whether there is a common process underlying the enhanced recency effects found in the auditory and the moving-box conditions. When the recency score is used as the dependent variable, the significant (and consistent) correlation between these conditions has suggested an affirmative answer. However, there has been little to suggest that the relationship between the two conditions is specific to the recency portion of the serial-position curve. One way to examine the specificity of the relationship is to perform a similar analysis at another serial position. Analogous to the recency score, a primacy score could be formed by partialing recall at Serial Position 2 from recall at Serial Position 1. Unfortunately, this score would likely lead to no significant (or at least few consistent) correlations. The problem is that recall at Serial Position 1 and recall at Serial Position 2 are likely to reflect similar processes (e.g., amount of rehearsal; see Glenberg et al., 1980). To the extent that this is correct, partialing recall at Serial Position 2 from recall at Serial Position 1 removes much of the interesting (and stable) variance. Thus, this primacy score would primarily reflect noise in the data and would result in inconsistent correlations. In fact, when this score was formed, four pairs of conditions resulted in significant correlations, but none were consistent (none found in more than one experiment).

To overcome this problem, a primacy score was formed by partialing from Serial Position 1 recall at Serial Position 7 . This procedure should remove contributions of general level of performance; however, if the recency ef- 
fect and the primacy effect reflect substantially different processes, much of the stable variance should be left in these primacy scores. Using these primacy scores, three correlations were consistent across the experiments. The correlations between the auditory and orthographic conditions were $.49, .35$, and .38 in Experiments 1, 2, and 3 , respectively. Apparently, the same cognitive process underlies the primacy effect in the auditory and orthographic conditions, and it seems likely that this process is some form of verbal rehearsal. The correlations between the auditory and the stationary-box conditions were $.53, .34$, and .21 (n.s.); the correlations between the stationary-box and the moving-box conditions were .53, .33 , and .04 (n.s.). Although not as consistent across experiments, these correlations may also reflect common process contributing to primacy effects.

Interestingly, for the primacy scores, the correlations between the auditory and the moving-box conditions were $.23, .09$, and $-.37(p=.068)$. That is, the consistency noted for the recency scores (in the auditory and movingbox conditions) is notably absent for the primacy scores. Thus, it seems safe to conclude that the underlying process in common between the auditory and moving-box conditions is specific to the recency portion of the serialposition curve.

\section{EXPERIMENT 4}

The data from Experiments 1-3 lead to the conclusion that the recency effects found in the auditory and movingbox conditions are produced by the same underlying process that is sensitive to dynamic changes in the stimuli. However, there is an alternative interpretation. In all three experiments, the consistent correlation in the recency scores was found between the conditions having the largest recency effects (auditory and moving box). Might the correlation be an artifact of the size of the recency effects? Experiment 4 tested this alternative. Subjects freely recalled auditory stimuli, orthographic stimuli, and the names of pictorial stimuli. All three of these conditions should produce substantially larger recency effects than those found in the serial recall of the smallvocabulary lists used in Experiments 1-3. If the correlation between the recency scores is an artifact of the size of the recency effect, then we should observe many correlations among the conditions. On the other hand, if the correlations observed in Experiments 1-3 reflect a process based on dynamic stimulus information, then the auditory recency scores should not correlate highly with either the orthographic or the pictorial recency scores.

\section{Method}

Subjects. A total of 36 subjects (10 men, 26 women) participated in the experiment.

Materials and Design. The to-be-recalled materials consisted of 216 items selected from the Snodgrass and Vanderwart (1980) norms. The items were those with the highest name agreement be tween subjects. The items were randomly divided into 1812 -item lists. Three versions of each list were created. One version con- sisted of slides of the drawings of each item (picture lists), a second version consisted of slides of the names of each drawing (orthographic lists), and the third version was a recording of the spoken names (auditory lists). Each picture list and each orthographic list was preceded by a slide displaying a row of asterisks as a ready signal and ended with a slide displaying a row of question marks as a recall signal. Auditory lists were preceded by a recording of the spoken word "ready" and followed by a recording of a tone, which was the recall signal.

Each subject studied and freely recalled six picture lists, six orthographic lists, and six auditory lists. The six lists in each presentation condition were blocked. The order of the blocks was counterbalanced over subjects. Within each block, half of the subjects were presented the lists in the order 1-6; the other subjects were presented the lists in the order 4-6, 1-3. In addition, all items served equally often in picture lists, orthographic lists, and auditory lists.

Procedure. The subjects participated individually. After hearing general instructions, each block of lists was preceded by specific instructions concerning the modality of presentation, ready signal, and recall signal. In all cases, each item was presented for $1.5 \mathrm{sec}$ (including the ready and recall signals). The subjects were given $90 \mathrm{sec}$ for written free recall immediately following the recall signal for each list.

\section{Results}

Mean recall (out of a possible six) is displayed in Figure 4. The main effect for presentation condition was significant $\left[F(2,70)=4.26, M S_{\mathrm{e}}=1.99\right]$. The means were 3.67 for the auditory lists, 3.56 for the pictures lists, and 3.39 for the orthographic lists. Although the difference between pictures and orthographic presentation in mean recall was not significant $\left[F(1,35)=2.29, M S_{\mathrm{e}}=\right.$ 2.77 ], recall of pictures did exceed recall of the words in 9 of the 12 serial positions, providing some replication of the standard picture-word superiority effect (Paivio, 1986). The main effect of serial position was significant $\left[F(11,385)=42.87, M S_{\mathrm{e}}=2.00\right]$, as was the interaction of presentation condition and serial position $[F(22,770)=$ $\left.3.10, M S_{\mathrm{c}}=1.21\right]$.

The modality effect is an enhanced recency effect, relative to that found in the orthographic condition. In Experiments 1-3, the recency effect was measured by the difference in recall between the last and second-to-last positions. Using that definition in Experiment 4 resulted in an enhanced recency effect for the auditory condition, relative to the orthographic condition $\left[F(1,35)=3.80, M S_{\mathrm{e}}=\right.$ $.81, p=.06]$, but there was less evidence for an enhanced recency effect for the picture condition, relative to the orthographic condition $\left[F(1,35)=2.44, M S_{\mathrm{e}}=.82, p=\right.$ .13]. Judging from Figure 4, it appears that a better measure of the recency effect for these data would take into account recall in the last four positions. In particular, the linear contrast over the last four positions seems to accurately capture the notion of recency (see also, Glenberg, Bradley, Kraus, \& Renzaglia, 1983, for another use of the linear contrast as a measure of recency). With this measure, there was evidence for an enhanced recency effect for both the auditory and picture conditions. That is, the linear contrast for the auditory lists was significantly greater than was the contrast for the orthographic lists $[F(1,35)$ $\left.=10.64, M S_{\mathrm{e}}=.90\right]$, and the linear contrast for the pic- 


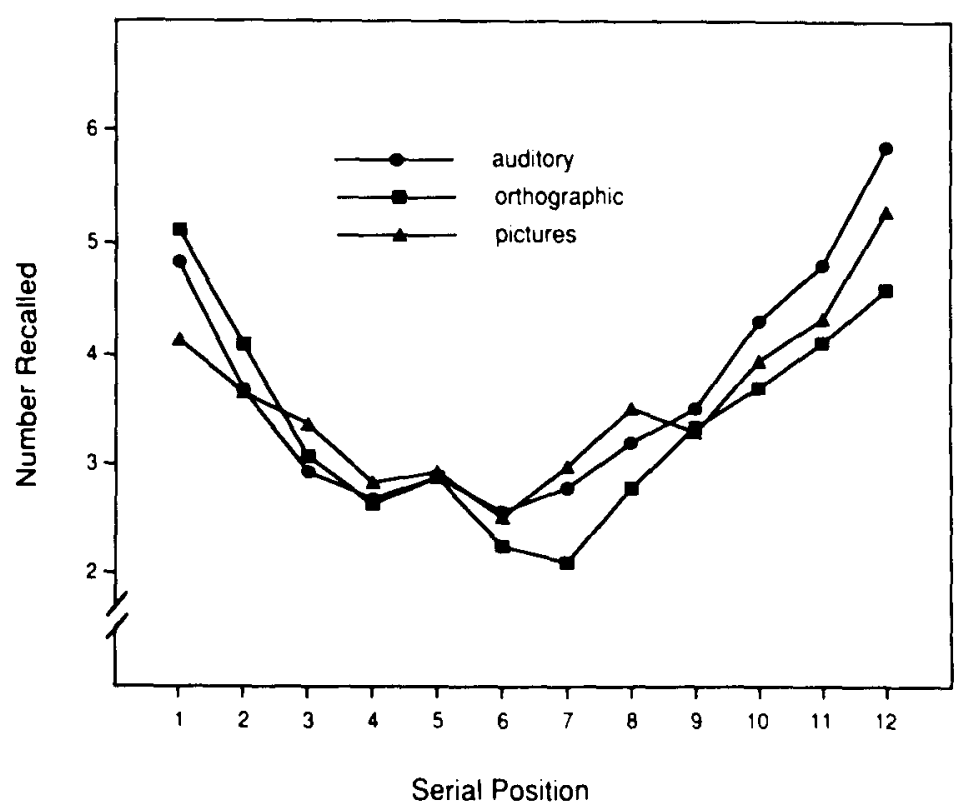

Figure 4. Number recalled from each serial position in Experiment 4.

ture lists was significantly greater than was the contrast for the orthographic lists $\left[F(1,35)=4.40, M S_{\mathrm{e}}=.89\right]$.

Now, the question is whether these large recency effects will produce significant correlations. Two scores were computed for each subject in each condition. These scores were the linear contrast over the last four positions and the sum of recall in the middle positions (Positions 5-8). Recall from the middle positions was then partialed from the linear contrasts. As in the previous experiments, the residual score will be referred to as the recency score (the recency effect over and above what can be predicted by performance in the middle of the list).

The correlations among the recency scores are presented in Table 4. There was a significant correlation between the picture and orthographic recency scores; however, neither the picture nor the orthographic recency scores correlated significantly with the auditory recency scores. Apparently, large recency effects are not sufficient to produce significant correlations among the recency scores.

Two additional analyses of recency scores were performed. In one, the recency scores were formed by partialing the sum of recall in the middle positions from the sum of recall in Positions 9-12 (as opposed to the linear contrast for these positions). In the second, the recency scores were formed by partialing the sum of recall in Serial Positions 5-8 from the sum of recall in Positions

Table 4

Correlations Among the Recency Scores in Experiment 4

\begin{tabular}{lccc} 
& Auditory & Orthographic & Pictures \\
\hline Auditory & .26 & .12 \\
Orthographic & & $.47^{*}$ \\
\hline${ }^{*} p<.05$ & & &
\end{tabular}

10-12. Both of these analyses produced patterns of significant and nonsignificant correlations identical to those in Table 4.

The results from these analyses are clear enough: Drawings of objects do produce an enhanced recency effect, but that enhanced effect is not strongly related to the recency effect found with auditory presentation. Again, the data are consistent with the claim that the auditory recency effect is due, at least in part, to processes sensitive to dynamic information.

Finally, several analyses of primacy scores were conducted. In the basic analysis, the primacy scores were formed by summing recall in Serial Positions 1-3 and partialing from this sum the sum of recall in Serial Positions 9-12 (similar to the most informative analyses of primacy scores for Experiments 1-3). Correlations among these scores were computed, and they are reported in Table 5. Note that the pattern of correlations for the primacy scores is quite different from the pattern of correlations for the recency scores (Table 4). In particular, the auditory primacy scores correlate with both the orthographic and the picture primacy scores. As for Experiments 1-3, a plausible explanation is that the primacy effect (as measured by the primacy scores) reflects a verbal rehearsal process, which is commonly applied regardless of the input modality.

A second primacy score was formed by partialing from the sum of Positions 1-3 the sum of Positions 5-8. The pattern of correlations was similar to that in Table 5, although all of the correlations were reduced (e.g., the correlation between the auditory and picture conditions was reduced to .32). Primacy scores were also formed by constructing the linear contrast across Positions 1-3 and partialing from the contrast various indices of mid- 
Table 5

Correlations Among the Primacy Scores in Experiment 5

\begin{tabular}{lccc} 
& Auditory & Orthographic & Pictures \\
\hline Auditory & $.57^{*}$ & $.41^{*}$ \\
Orthographic & & .27
\end{tabular}

${ }^{*} p<.05$

dle and recency effects. These various primacy scores also produced patterns similar to the pattern in Table 5 .

\section{GENERAL DISCUSSION}

The results of these experiments provide answers to the three questions posed in the introduction. First, Kallman and Cameron's (1989) effect can be replicated: When dynamic information is used in stimulus identification, there is an enhanced recency effect. In Experiment 1 , the recency effect for the moving-box condition was numerically greater than that for the other visual conditions, but the difference was not statistically significant. However, a statistically significant enhancement was found in Experiments 2 and 3 .

Second, the effect can be extended. In Experiment 3, speed of movement, rather than direction of movement, was the dynamic information used in identifying the stimulus, and this dynamic information engendered an enhanced recency effect. Furthermore, the results from Experiment 3 help to converge on the conclusion that it was the dynamic quality of the stimuli that was important. Because the to-be-remembered stimuli in Experiment 3 were terms referring to speed of movement rather than direction of movement (as in Kallman and Cameron and Experiments 1 and 2), we can be certain that use of direction terms is not a necessary component of the effect.

Given the enhancement associated with dynamic information, the third question is whether or not that enhancement can be attributed to the same processes as those that produce the enhanced recency effect with auditory presentation. There are many possible sources of recency effects, including recall based on a high-fidelity short-term or working memory (Atkinson \& Shiffrin, 1968), modalityspecific sensory registers (Crowder \& Morton, 1968), modality-specific coding (Nairne, 1988; Penney, 1989), grouping processes (Frankish, 1989), temporal distinctiveness (Glenberg \& Swanson, 1986), as well as processes based on dynamic qualities of stimuli (Campbell \& Dodd, 1980). Thus, it is not the case that auditory recency effects and recency effects produced by dynamic visual stimuli must be based on the same processes or mechanisms. In fact, as discussed shortly, the data indicate that there may well be multiple sources for recency effects.

The method used to decide if the same cognitive processes underlie multiple recency effects is based on an individual difference correlational analysis, using partialed scores (the recency score). As noted previously, the partialed score is preferred to the simple difference score for several psychometric reasons.
Across the experiments, there was one consistent correlation, the correlation between the auditory recency scores and the recency scores in the moving-box condition. These correlations were significant and numerically similar $(.40$, .43 , and .43 ) in the first three experiments. At the same time, the auditory recency scores never correlated significantly with the stationary-box recency scores, and the size of those correlations were numerically small (.15, -.02 , and .01 ). The results of Experiment 4 rule out the possibility that the significant correlations are an artifact of the size of the recency effects. Furthermore, the subsidiary analysis of the primacy scores indicates that the relationship between the auditory and moving-box conditions is specific to the recency portion of the serialposition curve. Thus, the evidence seems particularly strong that some process is contributing to both the auditory recency effect and the moving-box recency effect, but that process does not contribute to the stationary-box recency effect nor to primacy effects.

Now, what is that process? One possibility was suggested by Nairne (1988). He proposed that memory traces consist of two types of features, modality-independent and modality-dependent. According to Nairne, the enhanced auditory recency reflects the use of modality-dependent features in the reconstruction of information from traces. Furthermore, there is little visual (orthographic) recency effect because the visual modality-dependent features are overwritten by the postpresentation visual environment. Finally, Nairne suggests that movement produces modalitydependent features that are not overwritten by the postpresentation environment, leading to an enhanced recency effect in the moving-box condition.

While Nairne's account neatly explains the enhanced moving-box recency effect, by itself, it offers little explanation of when there is and when there is not a correlation between the recency scores. For example, one might grant the additional assumption that the correlation reflects the use of modality-dependent (either auditory or movementbased) features in reconstructing trace information. Note, however, there is also a recency effect in the stationarybox condition (see Figures 1-3), and yet those recency scores do not correlate with the auditory recency scores. Perhaps, one might assume further that the stationary-box recency effect, being no larger than the orthographic recency effect, is based on modality-independent features only, and, therefore, one would not expect it to correlate strongly with recall based on modality-dependent features. This argument runs afoul of the data from Experiment 4 (but to be fair, Nairne's account is meant to apply only to immediate serial recall, not free recall as in Experiment 4). In Experiment 4, the picture recency effect is enhanced, relative to the orthographic recency effect. Thus, according to Nairne's framework, the picture recency effect represents the use of modality-dependent features, and, by the first additional assumption given above, the picture recency scores should correlate with the auditory recency scores. However, they do not 
$(r=.12$; see Table 4). To summarize, Nairne's framework suggests an explanation for the enhanced recency effect found in the moving-box condition, but it will require further specification to account for the pattern of correlations among the recency scores.

An alternative account is that both the auditory and the moving-box recency effects reflect an enhancement based on perceptual grouping processes. In outline, the argument is as follows. An important (perhaps primary) function of perceptual processes is to deliver representations of objects in the environment, where objects are unified collections of properties, features, and parts. One clue that the perceptual system uses to construct objects is common dynamic changes among the components-that is, coherent movement signals objectness. Dynamic information in the auditory condition specifies the existence of a single object, a speaker, undergoing successive transformations. Dynamic information in the moving-box condition specifies the existence of a single object, the box, undergoing successive transformations. Finally, recall of information about a single object is superior to recall of information about multiple objects. The next few paragraphs flesh out this argument.

The case for objects has been made by Treisman (1988; Kahneman \& Treisman, 1984). She proposed that an object is represented by an "object file," a type of episodic memory trace that represents, initially, the perceptible characteristics of an object, such as its shape, location, and color. Perceptible changes in the object cause the object file to be updated (rather than the creation of a new object file), so that the identity of the object is maintained. Additional information (e.g., the object's name) may be added to the file from memory. Kahneman and Treisman adduce evidence indicating that the number of objects in a display (as opposed to the number of features) determines the perceptual load and that attention seems to be directed toward objects, rather than features. Thus, for example, Stroop interference (interference in naming the color of ink used to write a color name) is decreased if the to-benamed color and the color word are spatially separated, forming two objects. Also, some forms of repetition priming are enhanced when the prime and the target can be treated as the same object, rather than as mere physically identical instances of the same class (Treisman, 1988).

Common dynamic transformations among features are an important and powerful clue indicating that the features belong to the same object. In the visual domain, one illustration of this is the kinetic depth effect (Wallach, O'Connell, \& Neisser, 1953). When the shadow of a threedimensional object is projected onto a two-dimensional screen, it is difficult to determine the three-dimensional shape of the object from the shadow. However, when the object is rotated while casting a shadow, the threedimensional shape is readily apparent. Thus, dynamic information is used in constructing representations of objects. In fact, there is evidence to support the assertion that the information in dynamically changing optical arrays is the primary (ontogenetically prior) source of in- formation for maintaining a three-dimensional representation of the distal stimulus (Kellman, 1984).

In the auditory domain, stimuli with appropriate frequency and rhythmic relations are grouped into a unitary experience representing a particular auditory object, such as a musical instrument or a particular person's voice. These groups are called auditory streams (Bregman, 1981). Note that dynamic qualities of the stimuli, frequency and rhythm, contribute to grouping into perceptual objects.

The next step in the argument is that grouping facilitates memory in general and recall in particular. Kahneman and Treisman (1984) describe an experiment in which subjects viewed displays consisting of a word and a box with a gap on one side. The distance between the word and the gap was always the same, but, in one condition, the word appeared to be in the box and, in the other condition, the word appeared to be outside of the box. Following a mask, subjects were to report the word and the location of the gap. Recall (or identification) of the location of the gap was greater when the word and box formed a single object ( $84 \%$ correct) than when the word and box formed two separate objects ( $73 \%$ correct). Of course, that grouping (or chunking or organization) facilitates longer term memory performance is as close to an undisputed fact as can be found in the memory literature.

Importantly, a number of lines of evidence are converging on the conclusion that grouping processes, in addition to improving recall, underlie the auditory recency advantage. Frankish (1989) has demonstrated that grouping produced by temporal pauses during presentation facilitates recall of auditory items more than it facilitates recall of visual items. Penney (1989) reviewed evidence indicating that auditory items have automatic access to an "A code" that preserves temporal order. Glenberg and Fernandez (1988) and Greene and Crowder (1988) found that auditory presentation leads to more accurate temporal order judgments than does visual presentation. Given that one interpretation of memory for temporal order is that it is based on associative or grouping processes (Winograd \& Soloway, 1985), these results indicate enhanced grouping for auditory items, relative to visual items. A related line of evidence comes from the study of modality effects in rhythm-perception tasks. Glenberg, Mann, Altman, Forman, and Procise (1989) demonstrated that rhythms conveyed by auditory stimuli are easier to code than are rhythms conveyed by visual stimuli. Also, using a correlational analysis similar to the one used here, Glenberg, Mann, et al. demonstrated that the grouping processes involved in coding auditory rhythms are similar to the processes underlying the modality effect with verbal materials. Finally, Kahneman and Henik (1981) have suggested that suffix effects arise when the suffix is entered into the same perceptual group as the to-be-remembered stimuli.

The argument advanced so far is that dynamic information is used by both the auditory system and the visual system to form representations of objects and that recall 
of information from a single object exceeds recall of information from multiple objects. Given that both auditory and visual perception use dynamic information to identify stimuli, why is there an enhanced recency effect for auditory presentation, relative to the usual orthographic condition? In the typical memory experiment, the auditory stimuli can be perceptually grouped (streamed) to represent an object, the speaker. Easy access to the stimuli in the stream produces the recency effect. With orthographic presentation, the separate stimuli (words) do not form a unitary perceptual object; each word is its own object. Grouping of these separate objects requires intentional cognitive intervention (cf. Frankish, 1989).

In the moving-box condition, movement induces the perceptual system to treat the seven stimuli as instantiations of the same object: a box that moves from the center of the screen (in Experiments 1 and 2) toward the periphery. Furthermore, it is hypothesized that this identification results in grouping the stimuli such that the group represents seven successive transformations of the same object, just as the seven auditory stimuli are seven words spoken by the same speaker. In the stationary-box condition, the box makes discrete jumps from one location to another, unlike any real object. In this case, the perceptual system is offered few cues that would lead to grouping these stimuli. Thus, each box is treated as a separate perceptual object, much as with the orthographic stimuli.

The correlation between the auditory recency scores and the moving-box recency scores could arise in at least two ways. First, the correlation may represent constraints on grouping processes, constraints that are similar in the two modalities. Second, the correlation may reflect constraints on processes used to search or retrieve information from an organization.

This perceptual grouping account would seem capable of addressing the enhanced recency effects found with signed stimuli (Shand \& Klima, 1981), lip-read stimuli (Campbell \& Dodd, 1980), and mouthed stimuli (Nairne $\&$ Walters, 1983). In all of these cases, the perceptual system can use information to group the stimuli into a representation of a single object or a single object undergoing successive transformations. The account makes the following predictions. First, all of these types of enhanced recency effects should correlate with one another using analyses similar to those performed here. Second, all of these forms of recency effects should be disrupted by manipulations that force different grouping of the stimuli. For example, the recency effect for auditory words (or lip-read words, etc.) presented by different speakers should be reduced, relative to the effect for a single speaker. In a moving-box condition, the recency effect should be disrupted if the box changes (e.g., in color, size, or shape), so that the perceptual system no longer treats the stimuli as instantiations of the same object.

The explanation of the correlation between the auditory and moving-box recency scores is one issue. A related, but separate, issue is the number of sources of recency effects. If we trust the inferences based on the correlational analyses presented here, then we have evidence for multiple sources contributing to recency effects. One of these sources contributes to both auditory and moving-box recency effects. Another may contribute to orthographic and picture recency effects (see data in Table 4). Battacchi, Pelamatti, and Umiltà (1988) also concluded that there are multiple sources of recency effects, although they used the sort of dissociation logic that has been criticized by Dunn and Kirsner (1988). In any event, a contribution of the work reported here is that it describes a relatively easy-to-use correlational methodology for investigating hard questions about when similar-looking results are produced by the same underlying mechanisms.

\section{REFERENCES}

AtKinson, R. C., \& Shiffrin, R. M. (1968). Human memory: A proposed system and its control processes. In K. W. Spence \& J. T. Spence (Eds.), The psychology of learning and motivation (Vol. 2, pp. 89. 105). New York: Academic Press

Battacchi, M. W., Pelamatti, G. M., \& Umiltà, C. (1989). Multiple mechanisms for recency with vowels and consonants. Memory \& Cognition, 17, 329-336.

Bregman, A. S. (1981). Asking the "What For" question in auditory perception. In M. Kubovy \& J. R. Pomerantz (Eds.), Perceptual organization (pp. 99-118). Hillsdale, NJ: Erlbaum.

CAMpbell, R., DodD, B. (1980). Hearing by eye. Quarterly Journal of Experimental Psychology, 32, 85-99.

Chapman, L. J., \& Chapman, J. (1988). Artifactual and genuine relationships of laterality scores to accuracy. Psychological Bulletin, 104, $127-136$.

COHEN, J., \& COHEN, P. (1975). Applied multiple regression/correlation analysis for the behavioral sciences. Hillsdale, NJ: Erlbaum.

Crowder, R. G. (1982). A common basis for auditory sensory storage in perception and immediate memory. Perception \& Psychophysics, 31, 477-483.

Crowder, R. G. (1986). Auditory and temporal factors in the modality effect. Journal of Experimental Psychology: Learning, Memory, \& Cognition, 12, 268-278.

Crowder, R. G., \& Morton, J. (1969). Precategorical acoustic storage (PAS). Perception \& Psychophysics, 5, 365-377.

DUNN, J. C., \& KIRSNER, K. (1988). Discovering functionally independent mental processes: The principle of reversed association. Psychological Review, 95, 91-101.

FRANKISH, C. (1989). Perceptual organization and precategorical acoustic storage. Journal of Experimental Psychology: Learning, Memory, \& Cognition, 15, 469-479

Glenberg, A. M. Bradley, M. M., Kraus, T. A., \& Renzagla, G. J. (1983). Studies of the long-term recency effect: Support for a contextually guided retrieval hypothesis. Journal of Experimental Psychology: Learning, Memory, \& Cognition, 9, 231-255.

Glenberg, A. M., Bradley, M. M., Stevenson, J. A., Kraus, T. A., TKachuK, M. J., GretZ, A. L., Fish, J. H., \& Turpin, B. M. (1980). A two-process account of long-term serial position effects. Journal of Experimental Psychology: Human Learning \& Memory, 6, 355-369.

Glenderg, A. M., \& Fernandez, A. (1988). Evidence for auditory temporal distinctiveness: Modality effects in order and frequency judgments. Joumal of Experimental Psychology: Learning, Memory, \& Cognition, 14, 728-739.

Glenderg, A. M., Jona, M., \& Curran, T. (1989). Temporal coding in rhythm and recall tasks revealed by modality effects. Unpublished manuscript.

Glenberg, A. M., Mann, S., Altman, L., Forman, T., \& Procise, S. (1989). Modality effects in the coding and representation of rhythms. Memory \& Cognition, 17, 373-383. 
GlenberG, A. M. \& Swanson, N. C. (1986). A temporal distinctiveness theory of recency and modality effects. Joumal of Experimental Psychology: Learning, Memory, \& Cognition, 12, 3-15.

Greene, R. L., \& Crowder, R. G. (1988). Memory for serial position: Effects of spacing, vocalization, and stimulus suffixes. Journal of Experimental Psychology: Learning, Memory, \& Cognition, 14, 740-748.

Kahneman, K., Henik, A. (1981). Perceptual organization and attention. In M. Kubovy \& J. R. Pomerantz (Eds.), Perceptual organization (pp. 181-211). Hillsdale, NJ: Erlbaum.

Kahneman, K., Treisman, A. (1984). Changing views of attention and automaticity. In R. Parasuraman \& D. R. Davies (Eds.), Varieties of attention (pp. 29-61). Orlando, FL: Academic Press.

Kallman, H. J., \& Cameron, P. (1989). Enhanced recency effects with changing-state and primary-linguistic stimuli. Memory \& Cognition, 17, 318-328.

Kellman, P. J. (1984). Perception of three-dimensional form by human infants. Perception \& Psychophysics, 36, 353-358.

NaIRNE, J. S. (1988). A framework for interpreting recency effects in immediate serial recall. Memory \& Cognition, 16, 343-352.

NaIRNE, J. S., \& Walters, V. L. (1983). Silent mouthing produces modality-and suffix-like effects. Journal of Verbal Learning \& Verbal Behavior, 22, 475-483.

Paivio, A. (1986). Mental representations: A dual coding approach. New York: Oxford University Press.

Penney, C. G. (1975). Modality effects in short-term memory. Psychological Bulletin, 82, 68-84.

Penney, C. G. (1989). Modality effects and the structure of short-term verbal memory. Memory \& Cognition, 17, 398-422.
Shand, M. A., \& Kima, C. S. (1981). Nonauditory suffix effects in congenitally deaf signers of American Sign Language. Joumal of Experimental Psychology: Human Learning \& Memory, 7, 464-474.

SNODGRass, J. G., \& VANDERWART, M. (1980). A standardized set of 260 pictures: Norms for name agreement, image agreement, familiarity, and visual complexity. Journal of Experimental Psychology: $\mathrm{Hu}$ man Learning \& Memory, 6, 174-215.

Treisman, A. (1988). Features and objects: The fourteenth Bartlett memorial lecture. Quarterly Journal of Experimental Psychology, 40A, 201-237.

UNDERWOOD, B. J. (1975). Individual differences as a crucible in theory construction. American Psychologist, 30, 128-134.

Wallach, H., O'Connell, D. N., \& Neisser, U. (1953). The memory effect of visual perception of three-dimensional form. Journal of Experimental Psychology, 45, 360-368.

Winograd, E., \& Soloway, R. M. (1985). Reminding as a basis for temporal judgments. Journal of Experimental Psychology: Learning, Memory, \& Cognition, 11, 262-271.

\section{NOTES}

1. In fact, I came upon this possibility while running myself in the experiment. During the moving-box condition, I began, unintentionally, to whisper the names of the directions.

2. Thanks are due James Nairne for making this suggestion.

(Manuscript received January 29, 1990; revision accepted for publication May 23, 1990.) 